\title{
Prevalencia y caracterización genotípica de cepas de Staphylococcus aureus resistente a meticilina aisladas en un hospital regional mexicano
}

\author{
Prevalence and genotypic characterization of methicillin-resistant strains of Staphylococcus \\ aureus isolated in a Mexican regional hospital
}

\author{
Rosa M. Martínez-Medina' ${ }^{1}$ Fernando D. Montalvo-Sandoval', Martín Magaña-Aquino², Yolanda Terán-Figueroa ${ }^{3}$ y José T. Pérez-Urizar ${ }^{1}$
}

\author{
${ }^{1}$ Facultad de Ciencias Químicas de la Universidad Autónoma de San Luis Potosí. \\ ${ }^{2}$ Hospital Central Dr. Ignacio Morones Prieto. \\ ${ }^{3}$ Facultad de Enfermería y Nutrición, Universidad Autónoma de San Luis Potosí. \\ Financiamiento: Fondo Mixto (FOMIX) CONACyT -SLP, número de proyecto FMSLP-2014-02-250277. \\ Conflictos de interés: los autores declaran que no hubo conflictos de interés.
}

Recibido: 21 de septiembre de 2018 / Aceptado: 28 de noviembre de 2019

\section{Resumen}

Introducción: Staphylococcus aureus es uno de los patógenos con mayor prevalencia en el mundo, asociado a una alta tasa de mortalidad y un rápido desarrollo de resistencia a los antimicrobianos. A pesar de su patogenicidad, su seguimiento epidemiológico en México es escaso. Objetivo: Analizar la epidemiología molecular local y determinar el origen clonal de cepas resistentes a meticilina (RM) aisladas de pacientes internados en el Hospital Central "Dr. Ignacio Morones Prieto". Métodos: Se llevó a cabo un estudio prospectivo de corte transversal, de julio a diciembre de 2016. La caracterización de las cepas se realizó mediante genotipificación Spa, la determinación por RPC punto final de la frecuencia de genes de virulencia específicos y su antibiograma. Resultados: A partir de estos datos, se obtuvo que la prevalencia de $S$. aureus RM fue de $25,7 \%$, destacando la presencia del tipo Spa t895 en 76\% de las cepas resistentes y un patrón similar de susceptibilidad a antimicrobianos. Conclusión: Los resultados de este estudio indican que la prevalencia regional de SARM no se ha modificado en los últimos 10 años y proporcionan información valiosa del origen clonal y los factores de virulencia de las cepas de $S$. aureus aisladas en la región.

Palabras clave: Epidemiología molecular; Staphylococcus aureus; resistencia a meticilina; tipificación Spa.

\begin{abstract}
Background: Staphylococcus aureus is one of most prevalent pathogens in the world associated with a high mortality rate and a rapid development of resistance to antibiotics. Despite its pathogenicity, epidemiological monitoring in Mexico is scarce. Aim: To analyze the local molecular epidemiology and determine the clonal origin of methicillin-resistant (MR) strains isolated from patients admitted to Hospital "Dr. Ignacio Morones Prieto". Methods: A cross-sectional prospective study was carried out from July to December 2016. The characterization of the strains was carried out by Spa genotyping, frequency of specific virulence genes by PCR and antibiogram. Results: The prevalence of MRSA was $25.7 \%$, highlighting the presence of the Spa type 1895 in $76 \%$ of the resistant strains and a similar pattern of susceptibility to antibiotics. Conclusion: The results of this study indicate that the regional prevalence of MRSA has not changed in the last 10 years and provide valuable information on the clonal origin and the virulence factors of the strains of $S$. aureus isolated in the region.

Keywords: Molecular epidemiology; Staphylococcus aureus; methicillin resistance; Spa typing.
\end{abstract}




\section{Introducción}

$\circlearrowleft$ taphylococcus aureus es considerado universalmente como la principal causa de infecciones bacterianas en piel, tejidos blandos y hueso ${ }^{1}$ y una de las causas más comunes de bacteriemia asociada a hospitalización médica, puesto que su elevada patogenicidad se debe a una combinación de factores tales como su alta tasa de transferencia génica horizontal ${ }^{2}$, resistencia múltiple a antimicrobianos, así como su rápida adaptación y la producción de factores de virulencia ${ }^{3}$.

Dentro de los principales factores de virulencia se encuentran factores de adhesión como el factor de aglutinación, las proteínas de unión al fibrinógeno, la fibronectina y la sialoproteína ósea ${ }^{4}$, la formación de biopelículas que le confiere persistencia en el hospedero, la proteína A, citotoxinas y proteínas de adhesión extracelular, las que le permiten evadir los mecanismos de defensa del hospedero; además de la producción de proteasas, lipasas, nucleasas, fosfolipasa $\mathrm{C}$, hialuronidasas y elastasas implicadas en la destrucción de tejidos. Los factores antes mencionados, sumados a una elevada producción de toxinas, le confieren a $S$. aureus una gran capacidad de colonización e invasión tisular $^{4,5}$.

Por otra parte, S. aureus resistente a meticilina (SARM) es un patógeno común en pacientes hospitalizados, desde el desarrollo de meticilina en 1961, su incidencia ha ido en aumento tanto en el ámbito hospitalario como en la comunidad 6 . Un estudio realizado en Estados Unidos de América (E.U.A.) entre 1999 y 2005, mostró que los casos hospitalarios relacionados con $S$. aureus habían aumentado en $62 \%$ mientras que, en el mismo período, los casos relacionados con SARM aumentaron en $119 \%$, incrementando por lo tanto el porcentaje de aislados resistentes de 43 a $58 \%{ }^{7}$.

Debido a que las infecciones por SARM se detectaron inicialmente sólo en pacientes intrahospitalarios, se denominaron SARM-IH o HA-MRSA (por sus siglas en inglés hospital acquired MRSA); sin embargo, durante los años noventa se observó un incremento importante en el número de casos, principalmente en E.U.A. y Australia ${ }^{8}$ donde se detectaron además los primeros casos de infecciones por SARM adquiridas en la comunidad: SARM-AC o CA-SARM (por sus siglas en inglés community acquired $M R S A)^{9}$. Dado lo anterior, las cepas nosocomiales de SARM son microbiológica y genéticamente diferentes a las adquiridas en la comunidad, debido a la presencia de factores específicos de virulencia en estas últimas, como la exotoxina leucocidina de Panton-Valentine (LPV), una toxina citolítica que propicia infecciones graves de la piel y tejidos blandos, sepsis y neumonía necrosante ${ }^{10}$.

La resistencia a meticilina es conferida por la producción de una proteína de unión a penicilina de baja afinidad a antimicrobianos $\beta$-lactámicos (PBP2a) codificada, a su vez, por el gen $m e c A$. Éste se encuentra en una sección móvil del cromosoma de $S$. aureus conocida como casete cromosómico estafilocócico mec (sigla en inglés $\mathrm{SCC} m e c$ ), un elemento muy diverso de tamaño variable (tipos I a XI) y cuya longitud está relacionada con el origen clonal de las cepas. Mientras que los tipos I a III se han asociado a cepas intrahospitalarias, las de origen comunitario suelen acarrear los complejos tipo IV o $\mathrm{V}^{11}$.

La amplia variación de cepas de $S$. aureus ha llevado al desarrollo de métodos de tipificación basados en sus características fenotípicas y genotípicas, que permiten el monitoreo de la distribución de los principales clones a nivel mundial, destacando la tipificación Spa. Esta técnica se basa en el análisis de las repeticiones polimórficas en tándem de la región $\mathrm{X}$ variable del gen spa que codifica para la proteína A, propia de $S$. aureus. Esta región está integrada por 2 a 16 repeticiones en tándem de 21 a $24 \mathrm{pb}$ cada una. Con base en la amplificación y secuenciación de esta región, se han estandarizado dos nomenclaturas, la propuesta por Kreiswrirth que asigna una letra y un número a cada repetición, y la nomenclatura de RidomHarmsen, la más común e internacionalmente aceptada, que asigna un valor numérico a cada repetición y una letra " $t$ " seguida de un número específico a cada perfil identificado de repeticiones ${ }^{12}$. Sin embargo, a pesar de que la tipificación Spa establece una clasificación universal con un alto poder discriminatorio para diferenciar los distintos tipos de cepas y una reproducibilidad de $100 \%$, la asignación no siempre es representativa de las clasificaciones realizadas por otros métodos ${ }^{13}$.

No obstante, aunque México tiene sistemas robustos de vigilancia epidemiológica, no cuenta con organismos dedicados al control y registro de casos asociados con infecciones graves causadas por cepas de $S$. aureus, como en otros países ${ }^{14}$. Por lo tanto, el objetivo de este trabajo fue caracterizar cepas aisladas de pacientes en un hospital mexicano de segundo nivel de importancia en la región, mediante la identificación de genes que codifican para diversos factores de virulencia y resistencia, así como determinar la prevalencia y el origen clonal de las cepas mediante la tipificación Spa, para contribuir al conocimiento de la epidemiología molecular local de $S$. aureus.

\section{Materiales y Métodos}

Cepas. Staphylococcus aureus fue aislado e identificado a partir de cultivos de secreción (infecciones en piel y tejidos blandos e infecciones de herida quirúrgica), aspirado traqueal, exudado nasal, biopsia o infección por inserción de catéter, pertenecientes a casos clínicos ingresados en el Hospital Central "Dr. Ignacio Morones Prieto” en San Luis Potosí, México. El período de muestreo abarcó de julio a diciembre de 2016. 
Los cultivos duplicados fueron excluidos. La identificación y el antibiograma se realizaron mediante el sistema automatizado Vitek ${ }^{\circledR} 2$ (BioMérieux, Francia). Adicionalmente, se confirmó la identificación de la especie mediante la resiembra en el medio selectivo Vogel Johnson; la amplificación mediante reacción de polimerasa en cadena (RPC) del gen que codifica para la subunidad 23S ARNr y el gen $n u c$, específico de $S$. aureus; y, en el caso de las cepas resistentes a meticilina, tinción de Gram, la prueba de la coagulasa en tubo y la identificación por fermentación del agar de sal y manitol.

\section{Obtención de ADN}

El ADN genómico se aisló de cultivos "overnight" de acuerdo con el protocolo del kit comercial "Bacteria DNA Preparation Kit" (Jena Bioscience, Alemania); posteriormente, el $\mathrm{ADN}$ se almacenó a $-20^{\circ} \mathrm{C}$ hasta su análisis. La cuantificación de ADN se realizó en el equipo NanoDrop-1000 (Thermo Fisher Scientific, E.U.A.).

\section{Amplificación de genes de virulencia y resistencia}

Se empleó RPC punto final para identificar ocho genes relacionados con la virulencia de $S$. aureus, los genes de identificación de la especie (23S ARNr y nuc) y el gen de resistencia mecA (Tabla 1). Los factores de virulencia de importancia para los fines del estudio, así como las condiciones de amplificación y las secuencias de oligonucleótidos se seleccionaron con base en trabajos realizados en México con anterioridad ${ }^{3,15-17}$. La amplificación se llevó a cabo en un termociclador SimplyAmp mediante el uso de Red Load Taq Master Mix (Jena Bioscience, Alemania). Cada protocolo de amplificación se realizó en presencia de un control positivo $(S$. aureus ATCC 43300). Los productos amplificados se identificaron por electroforesis en gel de agarosa en tampón TBE con el marcador fluorescente EvaGreen ${ }^{\circledR}$ (Jena Bioscience, Alemania).

\section{Genotipificación Spa}

Los oligonucleóticos: 5'-CAA GCA CCA AAA GAG GAA-3' y 5'-CAC CAG GTT TAA CGA CAT-3' se utilizaron para la amplificación del gen spa-X [21]. Posteriormente, los productos de RPC se purificaron mediante

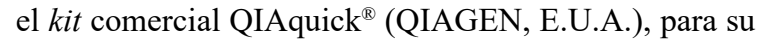
secuenciación en ambas direcciones en el Laboratorio Nacional de Biotecnología Agrícola, Médica y Ambiental (LANBAMA), (IPICyT, México). Finalmente, se utilizó el software BioNumerics ${ }^{\circledR} 7.6$ (Applied Maths, Bélgica) para el análisis de la región amplificada, determinación de repeticiones y tipos Spa. Adicionalmente, se generaron redes de agrupamiento filogenético a través del algoritmo UPGMA (Unweighted Pair Group Method using Arithmetic averages) y del árbol de expansión mínima (Minimum Spanning Tree).

\begin{tabular}{|ccl|}
\hline \multicolumn{2}{|c|}{ Tabla 1. Factores de virulencia y genes específicos estudiados } \\
\hline Gen & Tamaño del amplicón (pb) & Proteína codificada \\
\hline 23 rRNA & 1251 & Subunidad 23S rRNA \\
spa & Polimórfico & Proteína A de S. aureus \\
mecA & 310 & Proteína de unión a penicilina (PBP2A) \\
nuc & 279 & Termonucleasa estafilocócica \\
clfA & Polimórfico & Factor de agregación (ClfA) \\
IffB & 404 & Factor de agregación (ClfB) \\
cna & 452 & Adhesina de unión al colágeno (Cna) \\
bbp & 500 & Proteína de unión a sialoproteína ósea (Bbp) \\
ebpS & 550 & Proteína de unión a elastina de S. aureus (EbpS) \\
fnbA & 1279 & Proteína de unión a fibronectina A (FnBPA) \\
fnbB & 471 & Proteína de unión a fibronectina B (FnBPA) \\
LPV & 894 & Leucocidina de Panton-Valentine (PVL) \\
\hline
\end{tabular}

\section{Resultados}

Prevalencia de SARM. Se recolectaron 68 cepas de $S$. aureus, las que fueron identificadas en el hospital mediante el sistema Vitek ${ }^{\circledR} 2$. Sin embargo, sólo 66 fueron confirmadas como $S$. aureus por pruebas bioquímicas y por la amplificación del gen $23 \mathrm{~S}$ rRNA. De las cepas confirmadas, $61 \%$ provino de cultivos de secreción, $20 \%$ de aspirado traqueal, $10 \%$ de biopsia, $3 \%$ de punta de catéter, $3 \%$ de exudado nasal y $3 \%$ de hemocultivos con infección asociada al catéter (Tabla 2).

Se identificaron 49 cepas sensibles (CIM a oxacilina $<0,25 \mu \mathrm{g} / \mathrm{mL})$ y $17(25,7 \%)$ con resistencia a meticilina (CIM a oxacilina $>4 \mu \mathrm{g} / \mathrm{mL}$ ). Por RPC se encontró la presencia del gen mec $A$ en una de las cepas sensibles y su ausencia en una cepa resistente, por lo que se catalogaron como un falso negativo y un falso positivo. Del total de las cepas $(n=66), 68 \%$ fueron de pacientes masculinos y $32 \%$ de pacientes femeninos, en los que se identificaron 12 y 5 cepas resistentes, respectivamente.

\begin{tabular}{lcccc|}
\hline Tabla 2. Tipo de muestra de los aislados & \multicolumn{3}{c|}{} \\
\hline Cultivo & \multicolumn{2}{c}{ SARM } & \multicolumn{2}{c|}{ Total } \\
& $\mathbf{n}$ & $\mathbf{( \% )}$ & $\mathbf{n} \quad(\%)$ \\
Secreciones & 11 & $(64,7)$ & 40 & $(60,6)$ \\
Aspirado traqueal & 2 & $(11,8)$ & 13 & $(19,7)$ \\
Biopsia & 3 & $(17,6)$ & 7 & $(10,6)$ \\
Exudado nasal & 0 & $(0)$ & 2 & $(3)$ \\
Inserción de catéter & 0 & $(0)$ & 2 & $(3)$ \\
Hemocultivo & 1 & $(5,9)$ & 2 & $(3)$ \\
Total & $17(100)$ & $66(100)$ \\
\hline
\end{tabular}




\begin{tabular}{|c|c|c|c|c|c|c|c|}
\hline Tipo Spa & \multicolumn{2}{|c|}{ t895 $(n=13)$} & \multirow{2}{*}{$\begin{array}{l}\text { t622 } \\
\text { Interp. }\end{array}$} & \multirow{2}{*}{$\begin{array}{l}\text { t9364 } \\
\text { Interp. }\end{array}$} & \multirow{2}{*}{$\begin{array}{c}\text { ST } \\
\text { Interp. }\end{array}$} & \multirow{2}{*}{$\begin{array}{c}\text { C50 } \\
\text { Interp. }\end{array}$} & \multirow{2}{*}{$\begin{array}{c}\text { PVL }(\mathbf{n}=2) \\
\text { Interp. }\end{array}$} \\
\hline Antimicrobiano & Interp. & $\%$ & & & & & \\
\hline Bencilpenicilina & $R$ & 100 & $R$ & $R$ & $R$ & $R$ & $R$ \\
\hline Clindamicina & $\mathrm{R}$ & 100 & $\mathrm{R}$ & S & $\mathrm{R}$ & $\mathrm{R}$ & S \\
\hline Eritromicina & $\mathrm{R}$ & 100 & $\mathrm{R}$ & $\mathrm{R}$ & $\mathrm{R}$ & $\mathrm{R}$ & s \\
\hline Levofloxacina & $\mathrm{R}$ & 100 & S & $\mathrm{R}$ & $\mathrm{R}$ & $R$ & S \\
\hline Moxifloxacina & $R$ & $46,2^{\mathrm{a}}$ & S & $R$ & $R$ & $\mathrm{R}$ & s \\
\hline Quinupristina/dalfopristina & S & 100 & S & S & S & S & S \\
\hline Rifampicina & s & 84,6 & S & S & S & S & S \\
\hline Tigeciclina & S & 100 & S & S & S & S & s \\
\hline Vancomicina & s & 100 & S & S & S & S & s \\
\hline Ciprofloxacina & $\mathrm{R}$ & 100 & S & $\mathrm{R}$ & $\mathrm{R}$ & $\mathrm{R}$ & S \\
\hline Detección de resistencia a cefoxitina & + & 100 & + & - & + & + & - \\
\hline Gentamicina & s & 92,3 & S & S & S & S & S \\
\hline Linezolid & S & 100 & S & S & S & S & S \\
\hline Oxacilina & R & 100 & $\mathrm{R}$ & S & $\mathrm{R}$ & $\mathrm{R}$ & S \\
\hline Resistencia inducible a clindamicina & - & 100 & + & + & - & - & - \\
\hline Tetraciclina & S & 100 & S & S & S & S & S \\
\hline Cotrimoxazol & S & 100 & S & S & S & S & S \\
\hline
\end{tabular}

\section{Caracterización molecular}

Se identificaron 66 cepas positivas para clfA (100\%), 66 para $e b p S(100 \%), 57$ para $f n b A(86,4 \%), 56$ para $c l f B$ $(84,8 \%), 52$ para $b b p(78,8 \%), 42$ para $f n b B(63,6 \%)$ y 35 para cna (53\%). La combinación de genes: spa, clfA, $c l f B, f n b A$, fnbB, cna, $b b p$ y ebp $S$ se encontró más frecuentemente en cepas sensibles $(24,2 \%)$, mientras que sólo apareció en $6,1 \%$ de cepas resistentes, con una frecuencia total de 30,3\%. El amplicón correspondiente a LPV se encontró únicamente en dos aislados $(3,0 \%)$ correspondientes a cepas sensibles a meticilina con un antibiograma similar (Tabla 3). La tipificación del spa se completó en 54 cepas, 16 correspondientes a SARM y 38 a SASM. El tipo t895 (5' r26-r23-r17-r02-r17-r12-r17-r16 3', según la nomenclatura de repeticiones de Ridom-Harmsen) se presentó en 13 de las cepas resistentes, mientras que las restantes correspondieron a t622 (1), t9364 (1), un tipo nuevo sin asignación de número y una cepa (C50) cuya asignación de tipo fue inconclusa, pero muestra gran similitud con t895. En cuanto a las cepas sensibles, los tipos principales fueron t012 (4), t688 (3), t1 89 (3) y t148 (3). Las Figuras 1 y 2 muestran la relación entre las cepas analizadas por el dendrograma filogenético. Finalmente, se analizó el antibiograma de las cepas SARM, encontrando mínimas variaciones en el patrón de resistencia a los antimicrobianos evaluados entre las cepas t895 y una clara diferencia con los tipos t622 y t9364. Las cepas sin tipificación Spa mostraron un patrón de resistencia similar al presentado por el tipo t895 (Tabla 3).

\section{Discusión}

La prevalencia de SARM fue de $25,7 \%$, muy similar al $28 \%$ reportado en un estudio previo realizado en el año 2013 en este mismo hospital ${ }^{18}$, lo que demuestra que no ha habido cambios significativos respecto a la prevalencia de SARM-IH en los últimos cuatro años. Sin embargo, las diferencias encontradas pueden atribuirse a la estación del año en que se hizo la recolección, ya que se han documentado variaciones en la incidencia con respecto a los cambios estacionales ${ }^{19}$. Además, en el estudio previo mencionado, se encontró que los pacientes del sexo masculino tienen una probabilidad cuatro veces mayor de presentar infecciones por SARM, lo que coincide con la prevalencia observada en hombres $(70,5 \%)$ en nuestro estudio, por lo que sería altamente valioso evaluar posibles factores que determinan dicha tendencia.

$\mathrm{Al}$ comparar los datos de identificación obtenidos por RPC y aquellos proporcionados por el sistema Vitek ${ }^{\circledR} 2$ se encontró que dos cepas identificadas como $S$. aureus no 


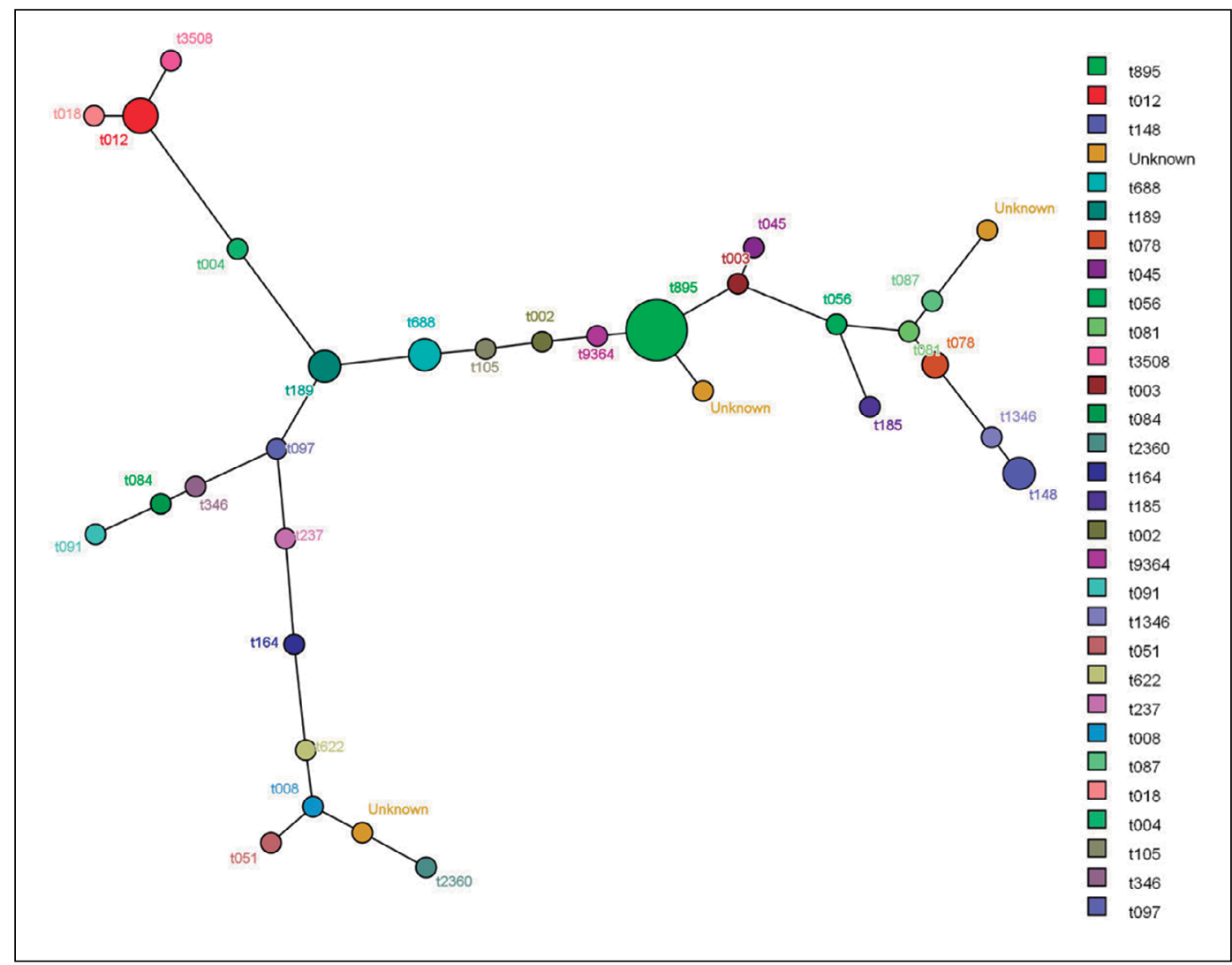

Figura 1. Árbol de expansión mínima o MST de 66 cepas de S. aureus. Cada conexión representa la cadena evolutiva más corta posible. Los nodos representan a cada tipo Spa, y el tamaño respectivo a la frecuencia con que se encuentran. El árbol muestra la relación entre todos los tipos sin distinción de resistencia a meticilina (elaborado en BioNumerics ${ }^{\circledR}$ 7.6).

presentaron el producto de RPC equivalente al esperado para el gen $23 \mathrm{~s}$ rRNA pero fueron positivas para $m e c A$. La tinción mostró la presencia de cocáceas grampositivas, mientras que las pruebas bioquímicas evidenciaron colonias de un tono rosáceo (en agar de sal y manitol) y fueron negativas para la prueba de la coagulasa. Es altamente probable que $S$. aureus haya estado presente en los cultivos originales, otorgando resultados positivos para Vitek ${ }^{\circledR} 2$, pero que $S$. epidermidis, u otro estafilococo estuviera presente con predominancia, debido a que este último es un comensal común de la piel y el segundo estafilococo más frecuentemente aislado en hospitales con una alta tasa de resistencia a meticilina $(80 \%)^{20,21}$, lo que explicaría los resultados obtenidos. En cualquier caso, y para fines estadísticos, no se consideraron a estas dos cepas para los resultados de prevalencia y caracterización.

Por otra parte, se encontró una discrepancia en la determinación de la resistencia a meticilina en dos cepas. Una de ellas identificada como sensible, mostró la amplificación del gen mec $A$ y presentó un tipo Spa t9364, el que muestra una similitud evolutiva mayor al $99 \%$ con el tipo 1895 asociado habitualmente con cepas SARM (Figura 2). Sin embargo, es muy probable que, a pesar de presentar el gen mecA, la expresión de PBP2a, pudo verse alterada por condiciones del crecimiento $\mathrm{u}$

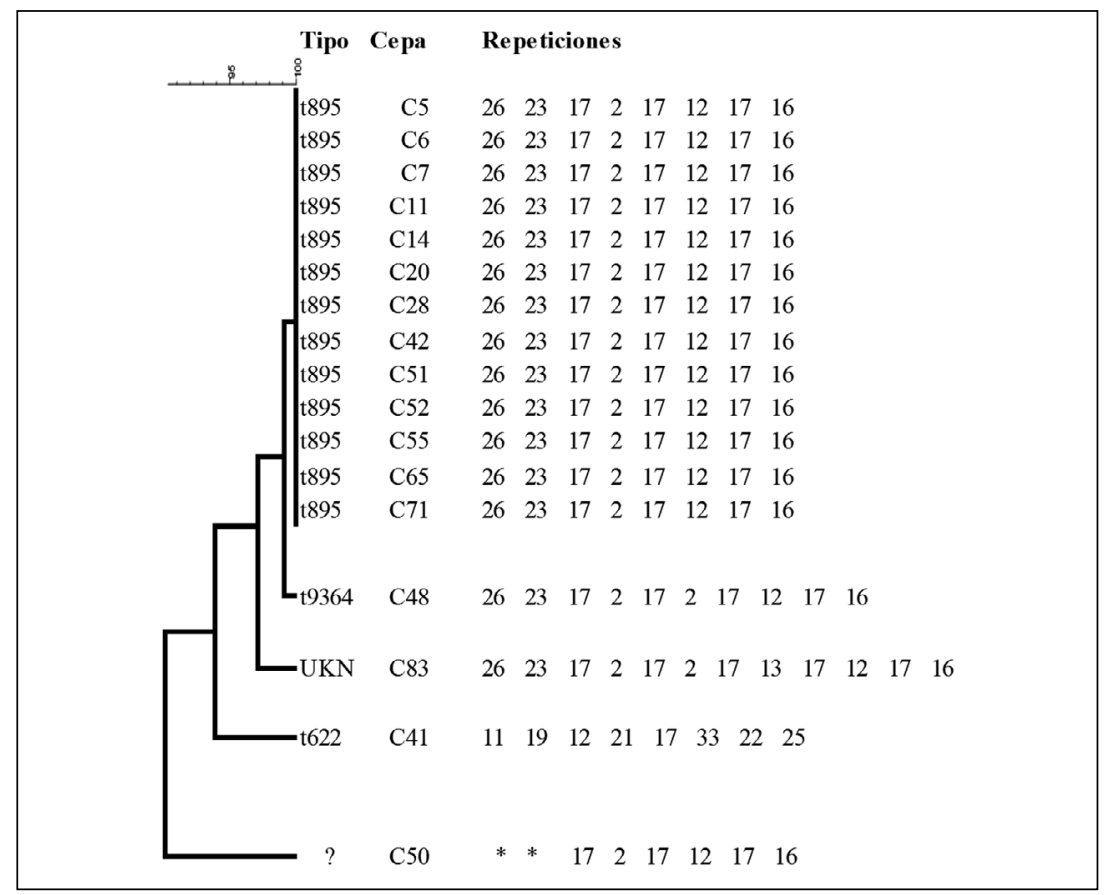

Figura 2. Dendrograma UPGMA. Izquierda: Distancias evolutivas entre cada tipo. Centro: Asignación de tipo Spa. Derecha: Serie de repeticiones correspondientes a cada cepa. * La secuencia de la cepa C50 no fue concluyente para las primeras repeticiones. 


\begin{tabular}{|c|c|c|c|c|c|c|}
\hline \multicolumn{6}{|c|}{ Genes (\%) } & \multirow{2}{*}{$\begin{array}{l}\text { Referencia } \\
\text { Nuestro estudio }\end{array}$} \\
\hline $\begin{array}{l}f n b A \\
f n b B \\
c l f B\end{array}$ & $\begin{array}{l}(86,4) \\
(63,6) \\
(84,8)\end{array}$ & $\begin{array}{l}\text { bbp } \\
\text { cna } \\
\text { ebps }\end{array}$ & $\begin{array}{l}(78,8) \\
(53) \\
(100)\end{array}$ & LPV & (3) & \\
\hline$f \cap b A$ & $(100)$ & $f \cap b B$ & $(100)$ & cna & $(100)$ & Arciola et al., $2005^{a}$ \\
\hline cna & $(38,40,56)$ & & & & & Foster \& Höök 1998ª \\
\hline $\begin{array}{l}e b p S \\
c l f B \\
b b p\end{array}$ & $\begin{array}{l}(85,4) \\
(81,8) \\
(78,2)\end{array}$ & $\begin{array}{l}\text { cna } \\
\text { fnbB } \\
\text { fnbA }\end{array}$ & $\begin{array}{l}(78,1) \\
(56,3) \\
(34,5)\end{array}$ & LPV & (0) & Paniagua-Contreras et al., $2012^{\mathrm{b}}$ \\
\hline $\begin{array}{l}c l f B \\
c n a \\
b b p\end{array}$ & $\begin{array}{l}(100) \\
(100) \\
(100)\end{array}$ & $\begin{array}{l}e b p S \\
\text { clfA } \\
\text { fnbA }\end{array}$ & $\begin{array}{l}(90,4) \\
(90,4) \\
(90,4)\end{array}$ & $f n b B$ & $(80,9)$ & Paniagua-Contreras et al., 2014ab \\
\hline $\begin{array}{l}f \cap b A \\
f n b B \\
c l f B\end{array}$ & $\begin{array}{l}(100) \\
(59,4) \\
(93,7)\end{array}$ & $\begin{array}{l}b b p \\
\text { cna }\end{array}$ & $\begin{array}{l}(87,5) \\
(93,7)\end{array}$ & $\begin{array}{l}\text { ebpS } \\
\text { LPV }\end{array}$ & $\begin{array}{l}(96,9) \\
(3,1)\end{array}$ & Paniagua-Contreras et al., 2014b \\
\hline$f \cap b A$ & $(100)$ & $f \cap b B$ & $(36,7)$ & cna & $(47,7)$ & Nashev et al., 2004 \\
\hline LPV & $(35,5)$ & LPV-SASM & $(51)$ & LPV-SARM & $(26,1)$ & Shrestha et al., 2014a \\
\hline $\begin{array}{l}\text { fnbA } \\
\text { clfB } \\
\text { cna }\end{array}$ & $\begin{array}{l}\left(89^{a}, 83^{b}\right) \\
(100) \\
\left(59^{a}, 75^{b}\right)\end{array}$ & $\begin{array}{l}b b p \\
e b p S \\
f n b B\end{array}$ & $\begin{array}{l}(100) \\
(100) \\
\left(95^{a}, 83^{b}\right)\end{array}$ & LPV & $\left(86^{a}, 75^{b}\right)$ & Campbell et al., 2008 \\
\hline $\begin{array}{l}\text { ClfA } \\
\text { clfB }\end{array}$ & $\begin{array}{l}(97,7) \\
(100)\end{array}$ & $\begin{array}{l}f n b B \\
f n b A\end{array}$ & $\begin{array}{l}(92,2) \\
(89,8)\end{array}$ & $\begin{array}{l}\text { ebpS } \\
\text { LPV }\end{array}$ & $\begin{array}{l}(68,8) \\
(19,5)\end{array}$ & Goudarzi et al., 2017b \\
\hline $\begin{array}{l}b b p \\
f \cap b A\end{array}$ & $\begin{array}{l}(0) \\
(96,23)\end{array}$ & $\begin{array}{l}\text { ClfB } \\
\text { cna }\end{array}$ & $\begin{array}{l}(43,3) \\
(11,3)\end{array}$ & $\begin{array}{l}e b p S \\
f n b B\end{array}$ & $\begin{array}{l}(9,3) \\
(1,8)\end{array}$ & Serray et al., 2016 \\
\hline
\end{tabular}

otros factores clave en la expresión de dicha proteína, proporcionando un fenotipo sensible. Mientras que, en la otra cepa que se identificó como resistente por Vitek ${ }^{\circledR}$ 2 , no se encontró la presencia del gen $m e c A$, por lo que se sugiere que esta cepa podría ser un caso de BORSA (Staphylococcus aureus con resistencia límite a oxacilina, en inglés: Borderline oxacillin-resistant Staphylococcus aureus), cuyo mecanismo de resistencia se basa en la sobre-producción de $\beta$-lactamasa estafilocócica, que degrada meticilina y oxacilina de manera apreciable, causando una resistencia límite con CIM de $2-4 \mu \mathrm{g} / \mathrm{ml}$ y 1-2 $\mu \mathrm{g} / \mathrm{ml}$, respectivamente ${ }^{22}$. El análisis de resistencia a cefoxitina por el método de Kirby-Bauer podría ayudar a confirmar el fenotipo de estas dos cepas ${ }^{23}$.

La combinación de genes que se encontró más frecuentemente en nuestro estudio es consistente con la combinación de genes encontrada con mayor frecuencia en cepas resistentes (24,2\%). En un estudio realizado en México en 2012, Paniagua-Contreras y cols., evaluaron la presencia de 35 genes que podrían estar relacionados con la colonización de catéteres, encontrando la combinación antes mencionada en $11 \%$ de sus cepas. Posteriormente, en 2014 analizaron algunos de estos genes observando que $47,7 \%$ de cepas SARM expresó la combinación spa, clf $A, c l f B, f n b A, f n b B, c n a, b b p$, ebps (además de eap, $s d r C, s d r D, S d r E$, efb, icaA y $a g r$ ). Al ser ésta la que se encontró en mayor proporción en las cepas evaluadas en nuestro estudio, se sugiere una gran prevalencia de cepas acarreando dicha combinación en las salas del hospital evaluado, y podría ser la causa de la similitud clonal observada en los resultados de SARM (Figura 2).

Proteínas de adhesión como la ClfA, ClfB, FnbA, FnbB y Cna están implicadas en la adherencia a células $\mathrm{y}$ tejidos en infecciones invasoras como osteomielitis, endocarditis infecciosa y artritis séptica. La mayoría de las cepas analizadas en este estudio fueron obtenidas de pacientes con endocarditis, infección ósea / articular, infección de piel y tejidos blandos o bacteriemia, por lo que los genes encontrados indican que estas proteínas pueden desempeñar un papel importante en las enfermedades clínicas causadas por $S$. aureus, a pesar de no tener una distribución significativamente diferente entre las diversas enfermedades. Varios estudios han evaluado la prevalencia de los principales genes que codifican factores de virulencia en $S$. aureus (Tabla 4) con resultados similares al presente estudio. Sin embargo, se pueden apreciar 
Un estudio de epidemiología molecular en 500 cepas de ocho países europeos asoció la clasificación Spa con el linaje clonal determinado mediante tipificación del casete cromosómico y MLST (en inglés, Multilocus Sequence Typing). En el reporte se obtuvo que los tipos Spa t002, t688 y t895 (n total $=8)$ pertenecen al linaje ST5 con SCCmec tipo II (LPV- $)^{29}$. Estos tipos fueron también los encontrados en SARM en el presente estudio (t002 en SASM). Cabe destacar que el linaje ST5-MRSA-II corresponde a la clona Nueva York/Japón, de manera que los tipos Spa encontrados, incluyendo las características de las cepas, y las referencias en la literatura científica a la presencia de este linaje en México, permiten suponer la presencia y predominio de este clon en San Luis Potosí. Evidentemente esto se tiene que comprobar mediante la tipificación por otros métodos, como MLST, pero constituye una aproximación cercana a la realidad en un país que no cuenta con suficientes estudios epidemiológicos de $S$. aureus que lleven a un control adecuado de la dispersión de las cepas.

tejidos blandos, neumonía necrosante e infecciones ôs y articulares en humanos ${ }^{25}$. En 2016, Guillen y cols., observaron una mayor tendencia para presentar infecciones invasoras en pacientes con infecciones por cepas SASM-LPV positivas ${ }^{26}$. Mientras que un estudio realizado en España demostró que la presencia de LPV en cepas de $S$. aureus causantes de neumonía aumenta 1,5 veces el riesgo de muerte ${ }^{27}$. Respecto a nuestros resultados, las cepas positivas para LPV provenían de pacientes con infecciones invasoras (neumonía atípica e infección de pie diabético). Si bien los resultados de tipificación de estas dos cepas fueron inconclusos, no se puede descartar que se trate de cepas de origen comunitario (SARM-AC) puesto que, en 2010, se informó la presencia de una cepa US-300 de origen comunitario en el Hospital Central "Dr. Ignacio Morones Prieto"28, lo que indicaría que, aunque a baja frecuencia, la presencia de cepas resistentes en la comunidad podría haberse mantenido y sería relevante para el control epidemiológico de SARM en la región.

La clasificación Spa reveló el predominio del tipo $t 895$ en 13 cepas SARM, el que fue reportado anteriormente en México en $76 \%$ de los aislados ${ }^{16}$. El resto de los tipos encontrados muestran una similitud filogenética muy alta, así como una resistencia a múltiples antimicrobianos claramente diferenciada (Tabla 3), lo que ha sido observado en cepas resistentes a meticilina ${ }^{16}$. Aunque no se observó una relación entre las combinaciones de los factores de virulencia y las clonas, se distingue que estas cepas del tipo 1895 tienden a acarrear un mayor número de adhesinas. Adicional a este estudio, es primordial el análisis de los factores asociados a la formación de biopelículas y diversos procesos patológicos en los que la expresión conjunta de distintas adhesinas, toxinas y genes regulatorios pueda explicar el mecanismo de la infección, así como mejorar el entendimiento de la patogenicidad de las cepas.

\section{Conclusiones}

Nuestros resultados indican que la prevalencia de SARM es consistente con lo reportado en otras regiones de México y se mantiene en niveles semejantes a los reportados en años previos en el mismo hospital. Además, el análisis de caracterización molecular proporciona información significativa de los factores de virulencia y del origen clonal de las cepas con mayor prevalencia. A través de la inclusión de otros métodos de caracterización molecular (PFGE, SCCmec y MLST) se podría confirmar la presencia de estos clones en San Luis Potosí. El presente estudio proporciona nuevos datos de la epidemiología regional y nacional de $S$. aureus que se suman a los ya existentes y contribuyen a mejorar su control y vigilancia. De la misma manera, estos datos favorecen el diseño de políticas y estrategias terapéuticas más eficientes y rentables, al optimizar el uso de antimicrobianos de acuerdo con las características específicas del clon y prevenir así el desarrollo de cepas resistentes a múltiples antimicrobianos, fenómeno que en la actualidad amenaza drásticamente a los sistemas de salud.

Agradecimientos. Al Fondo Mixto (FOMIX) CONACyTSLP por los recursos aportados para el desarrollo de este estudio bajo el proyecto número FMSLP-2014-02-250277. En este trabajo se hizo uso del sitio web de tipificación Spa (http://www.spaserver.ridom.de/) desarrollado por Ridom GmbH y operado por SeqNet (http://www.SeqNet.org/). Agradecemos la licencia concedida por Applied Maths NV para el uso del software BioNumerics ${ }^{\circledR}$ 7.6. 


\section{Referencias bibliográficas}

1.- Dryden M S. Complicated skin and soft tissue infection. J Antimicrob Chemother 2010; 65: iii35-44. doi: 10.1093/jac/dkq302.

2.- Torres Escobar I. 2014. Estudio de la resistencia de Staphylococcus aureus meticilino resistente (MRSA) e identificación del gen $m e c A$ por reacción en cadena de la polimerasa en tiempo real empleando el equipo: GeneXpert Cepheid. Undergraduate thesis. Universidad Autónoma del Estado de México, México. https://pdfs. semanticscholar.org/24e4/9a8fcc3fadb6b85dec 5edec379f4388917a0.pdf.

3.- Paniagua Contreras G L, Monroy Pérez E, Vaca Pacheco S, González Almazán S E. Resistencia a antibióticos y metales pesados en cepas clínicas de Staphylococcus aureus. Rev Med Hosp Gen 2003; 66: 13-21. https://www. medigraphic.com/pdfs/h-gral/hg-2003/hg031c. pdf.

4.- Pahissa Berga A. Infecciones producidas por Staphylococcus aureus (1ed) Barcelona Marge Medica Books, Spain.

5.- Velazco Pizarro V C. 2014. Detección y tipificación molecular de Staphylococcus aureus y Staphylococcus aureus resistente a meticilina (SARM) en Dakota del Norte, Estados Unidos. PhD thesis. Universidad de Concepción, Chile. http://repositorio. udec.cl/jspui/bitstream/11594/905/1/ Tesis_Deteccion_y_Tipificacion_Molecular_ Staphylococcus.Image.Marked.pdf.

6.- Oliveira D C, Tomasz A, De Lencancastre, H. Secrets of success of a human pathogen: molecular evolution of pandemic clones of meticillin resistant Staphylococcus aureus. Lancet Infect Dis 2002; 2:180-9. doi 10.1016/ s1473-3099(02)00227-x.

7.- Klein E, Smith D L, Laxminarayan R. Hospitalizations and deaths caused by methicillin-resistant Staphylococcus aureus, United States, 1999-2005. Emerg Infect Dis 2007; 13: 1840-6. doi: 10.3201/ eid1312.070629.

8.- Jiménez Quiceno J N, Correa Ochoa M. M. Staphylococcus aureus resistente a meticilina: bases moleculares de la resistencia, epidemiología y tipificación. Iatreia 2009; 22: 147-58. https://www.redalyc.org/ pdf/1805/180513869006.pdf.

9.- Udo E, Pearman J, Grubb W. Genetic analysis of community isolates of methicillinresistant Staphylococcus aureus in Western Australia. J Hosp Infect, 1993; 25: 97-108. doi: 10.1016/0195-6701(93)90100-e.

10.- Shrestha B, Singh W, Raj V S, Pokhrel B M, Mohapatra T M. High prevalence of Panton-Valentine Leukocidin (PVL) genes in nosocomial-acquired Staphylococcus aureus isolated from tertiary care hospitals in Nepal. Biomed Res Int 2014: 1-7. http://dx.doi. org/10.1155/2014/790350.

11.- Kong E F, Johnson J K, Jabra-Rizk M A. Community-associated methicillin-resistant Staphylococcus aureus: an enemy amidst us.
PLoS Pathog, 2016; 12: e1005837. https://doi. org/10.1371/journal.ppat.1005837.

12.- Hallin M, Friedrich A W, Struelens M J. spa Typing for epidemiological surveillance of Staphylococcus aureus. Methods Mol Biol. 2009; 551: 189-202. doi: 10.1007/978-1-60327999-4_15.

13.- Koreen L, Ramaswamy S V, Graviss E A, Naidich S, Musser J M, Kreiswirth B N. spa typing method for discriminating among Staphylococcus aureus isolates: implications for use of a single marker to detect genetic micro-and macrovariation. J Clin Microbiol 2004; 42: 792-9. https://doi.org/10.1128/ jcm.42.2.792-799.2004.

14.- Miranda Novales M G. Resistencia antimicrobiana del Staphylococcus aureus en México. Bol Med Hosp Infant Mex 2011; 68: 262-70. https://www.medigraphic.com/pdfs/ bmhim/hi-2011/hi114b.pdf.

15.- Paniagua Contreras G, Sáinz Espuñes T, Monroy Pérez E, Rodríguez Moctezuma J R, Arenas Aranda D, et al. Virulence markers in Staphylococcus aureus strains isolated from hemodialysis catheters of Mexican patients. Adv Microbiol 2012; 2:476-87. doi: 10.4236/ aim.2012.24061.

16.- Paniagua-Contreras G L, Monroy-Pérez E, Vaca-Paniagua F, Rodríguez-Moctezuma J R, Negrete-Abascal E, Vaca S. Implementation of a novel in vitro model of infection of reconstituted human epithelium for expression of virulence genes in methicillin-resistant Staphylococcus aureus strains isolated from catheter-related infections in Mexico. Ann Clin Microbiol Antimicrob 2014; 13: 6. doi: 10.1186/1476-0711-13-6.

17.- Paniagua-Contreras G L, Monroy-Pérez E, Gutiérrez-Lucas R, Sainz-Espuñes T, BustosMartínez, Vaca S. Genotypic characterization of methicillin-resistant Staphylococcus aureus strains isolated from the anterior nares and catheter of ambulatory hemodialysis patients in Mexico. Folia Microbiol 2014; 59: 295-302 https://doi.org/10.1007/s12223-013-0300-4.

18.- Rivas Rangel A, González Castilla E, De Lira Torres M, Flores Santos A, Fragoso Morales L E. Pacientes de sexo masculino ¿Mayor susceptibilidad a infecciones por Staphylococcus aureus resistente a meticilina? Enf Inf Microbiol 2013; 34: 50-3. https://www. medigraphic.com/pdfs/micro/ei-2014/ei142b. pdf.

19.- Verón M T, Ojeda M G, Avino F, Spelzzini A, Barboza A L, Petrozzino, Y. Incidencia y distribución estacional de Staphylococcus aureus resistente a la meticilina en pacientes adultos ambulatorios en una clínica de la provincia de Buenos Aires: periodo 2006-2011. Rev Argent Microbiol 2012; 44: 306-11. https:// www.redalyc.org/pdf/2130/213025174012.pdf.

20.- García-Apac C, Pardo-Valdespino J, SeasRamos C. Bacteremia por Staphylococcus epidermidis y absceso de partes blandas en un paciente post-operado: reporte de un caso. Rev Med Hered 2003; 14: 221-3. http://www.scielo. org.pe/pdf/rmh/v14n4/v14n4cc01.pdf.

21.- Galdbart J O, Allignet J, Tung H S, Rydèn C, El Solh N. Screening for Staphylococcus epidermidis markers discriminating between skin-flora strains and those responsible for infections of joint prostheses. J Infect Dis 182: 351-5. doi: 10.1086/315660.

22.- Gil Diez-de Medina M. Staphylococcus aureus: Microbiología y aspectos moleculares de la resistencia a meticilina. Rev Chilena Infect 2000; 17: 145-52. https://scielo.conicyt.cl/pdf/ rci/v17n2/art10.pdf.

23.- Acosta-Pérez G, Rodríguez-Ábrego G, Longoria-Revilla E, Castro-Mussot M E. Evaluación de cuatro métodos para la detección de Staphylococcus aureus meticilino-resistente de muestras clínicas en un hospital regional. Salud Pública Mex 2012.54: 1-6. https://www. medigraphic.com/pdfs/salpubmex/sal-2012/ sal121a.pdf.

24.- Carpinelli L. Caracterización fenótípica y genotípica de Staphylococcus aureus aislados de trabajadores de la salud de un centro hospitalario de referencia nacional [Internet]. UNA. Dirección General de Postgrado. Dirección General de Investigación Científica y Tecnológica. IICS; 2011 [citado 24 de abril de 2019]. Disponible en: http://bases.bireme.br/ cgi-bin/wxislind.exe/iah/online/?IsisScript=iah/ iah. $x$ is $\& s r c=$ google $\&$ base $=$ LILACS\&lang $=p \&$ nextAction $=$ lnk\&exprSearch $=600322 \&$ indexSe $\operatorname{arch}=$ ID.

25.- Muttaiyah S, Coombs G, Pandey S, Reed P, Ritchie S, Lennon D, et al. Incidence, risk factors, and outcomes of Panton-Valentine Leukocidin-positive methicillin-susceptible Staphylococcus aureus infections in Auckland, New Zealand. J Clin Microbiol 2010; 48 (10): 3470-4. doi: 10.1128/JCM.00911-10.

26.- Guillén R, Carpinelli L, Rodríguez F, Castro H, Quínónez B, Campuzano A, et al. Staphylococcus aureus adquiridos en la comunidad: caracterización clínica, fenotípica y genotípica de aislados en niños paraguayos. Rev Chilena Infectol 2016; 33 (6): 609-18. https://scielo.conicyt.cl/pdf/rci/v33n6/art02.pdf.

27.- López-Aguilar C, Pérez-Roth E, MéndezÁlvarez S, Moreno A, Durán M, Casanova C, et al. Association between the presence of the Panton-Valentine leukocidin-encoding gene and a lower rate of survival among hospitalized pulmonary patients with staphylococcal disease. J Clin Microbiol 2007; 45 (1): $274-$ 276. doi: 10.1128/JCM.02032-06.

28.- Martínez-Aguilar G. Análisis de genotipos y de los tiempos de duplicación de cepas de Staphylococcus aureus resistente a meticilina aisladas de infecciones nosocomiales y adquiridas en la comunidad. $\mathrm{PhD}$ thesis. Universidad de Colima, México.

29.- Hetem D J, Derde L P, Empel J, Mroczkowska A, Orczykowska-Kotyna M, Kozinska A, et al. Molecular epidemiology of MRSA in 13 ICUs from eight European countries. J Antimicrob Chemother 2016; 71:45-52. doi: 10.1093/jac/ dkv298. 